\title{
Our World Isn't Organized into Levels
}

\author{
Angela Potochnik
}

\begin{abstract}
Levels of organization and their use in science have received increased philosophical attention of late, including challenges to the well-foundedness or widespread usefulness of levels concepts. One kind of response to these challenges has been to advocate a more precise and specific levels concept that is coherent and useful. Another kind of response has been to argue that the levels concept should be taken as a heuristic, to embrace its ambiguity and the possibility of exceptions as acceptable consequences of its usefulness. In this chapter, I suggest that each of these strategies faces its own attendant downsides, and that pursuit of both strategies (by different thinkers) compounds the difficulties. That both kinds of approaches are advocated is, I think, illustrative of the problems plaguing the concept of levels of organization. I end by suggesting that the invocation of levels may mislead scientific and philosophical investigations more than it informs them, so our use of the levels concept should be updated accordingly.
\end{abstract}

Levels of organization have featured prominently in a number of philosophical debates. They have also been invoked in a variety of scientific contexts, especially in biology, where textbooks tend to be organized with levels of organization as the organizing principle. In recent years, increased philosophical attention has been devoted to concepts of levels of organization and how these have been deployed in science and philosophy. Some of this work has been critical. Rueger and McGivern (2010) challenge whether the traditional 
conception of levels is apt in physics and propose an alternative. Potochnik and McGill (2012) aim to identify and challenge broad invocations of levels of organization that occur in both philosophy and biology. In our view, such invocations tended to anticipate a number of broad implications from levels that by and large fail to materialize and to rest on intuitions that, though widely shared, are difficult to justify philosophically. Eronen $(2013,2015)$ points out limitations to mechanistic conceptions of levels of organization in particular, which many see as the most promising levels concept currently on offer.

Some of these same discussions, as well as other work, have used the ambiguity of and associated difficulties with the broad, intuitive levels concept as an opportunity to refine the concept and how it is employed. Craver (2007) advocates a mechanistic conception of levels and distinguishes this from the more traditional conception of levels of organization based on compositional relationships of parts and wholes. Rueger and McGivern (2010) propose scale as a replacement for levels in physics. Potochnik and McGill (2012) also suggest the use of scale to articulate "quasi-levels" according to the bounds within which some causes tend to dominate. DiFrisco (2017) argues that temporal scale alone holds promise for achieving the goals of the invocation of levels. These are all attempts to disambiguate the levels concept or motivate a suitable substitute, giving the concept a precise, specific interpretation based on the relation used to articulate levels - composition, mechanism, spatial scale, or temporal scale. Some also explicitly attend to the relation used to group entities or processes onto the same level, which - as Eronen (2013) and DiFrisco (2017) make explicit-is a distinct question. I'll refer to this strategy as making the levels concept precise.

A different strategy has been to embrace the broad, intuitive levels concept, along with its limitations and difficulties. Many of our scientific concepts are imprecise and ambiguous. This has been shown to be so for the concept of the gene, for example, which might refer (at least) to a stretch of DNA or to the genetic basis for some identified physical or behavioral trait. (For a recent discussion of the ambiguous and changing meanings of the gene concept, 
see Rheinberger and Müller-Wille, 2017.) Moreover, many concepts are usefully applied in some scientific contexts but not others. It's been crucially important to identify the gene variants related to some genetic diseases, but for other diseases, we have not identified identifiable genetic causes, and there's no reason to expect we will. Finally, imprecise and ambiguous concepts, like that of the gene, can be fruitfully applied in different ways in different scientific contexts. Perhaps the levels concept is like the gene concept in these regards.

This seems to be the tack taken by Wimsatt's (1972; 2007) classic and deeply influential treatment of levels of organization. He emphasizes that levels aren't found across nature and that, though some phenomena display a remarkable convergence of different features of levels, there may still be exceptions. And then, Brooks (2017) encourages philosophers and scientists to attend to the ways in which the levels concept varies in different applications and to accept that variability rather than see it as a weakness of the concept. Brooks and Eronen (2018) advocate the heuristic use of the levels concept in structuring scientific problems. In his chapter in this volume, Brooks argues for a "doctrinal" use of levels as structuring education and inquiry in the field of biology. This view posits the broad significance of the levels concept at least in biology, while also tolerating variability in application and in precise meaning. These treatments of levels of organization refuse to make the levels concept precise, as they see its multiple relevances and breadth of applicability as an important strength of that concept. They instead accommodate criticisms of the levels concept by granting variability in its use and limitations to its usefulness. I'll refer to this strategy as endorsing the heuristic use of the levels concept.

Here I explore these two strategies to clarifying the levels concept. I suggest that while each of these strategies is initially plausible, each also encounters its own significant difficulties. A specific, precise levels concept turns out to be remarkably difficult to characterize. One or another precise concept of levels may be useful in certain constrained 
scientific contexts, but none should be expected to apply generally nor to preclude the usefulness of other related or opposed concepts. And then, heuristic uses of the levels concept may not prove to be of much scientific or philosophical value, and they also risk being taken too literally and may occlude the recognition of other, more productive heuristics. Moreover, the pursuit in philosophical and scientific literatures of both strategies - making levels precise and treating levels as a heuristic - compounds the difficulties facing each. Indeed, that both kinds of approaches are advocated is, I think, illustrative of the problems plaguing the concept of levels of organization. In Section 1, I evaluate the strategy of making the levels concept more precise; in Section 2, I evaluate the strategy of endorsing the heuristic use of the levels concept. In Section 3, I suggest that the invocation of levels can mislead scientific and philosophical investigations just as much as it informs them. The limitations and ambiguity of levels concepts should be recognized, organization into levels should not be presumed, and scientists and philosophers should actively try to cultivate alternative heuristics for the organization of our world.

\section{In Pursuit of a More Precise Levels Concept}

Philosophical and scientific discussions of levels of organization have variously considered a number of different relations as relevant to levels. Classically, in what has been called

the "layer-cake" view of levels developed by Oppenheim and Putnam (1958), compositional relationships among parts and wholes are the basis for levels of organization in our world. In his seminal work on complexity, Simon (1962) emphasizes the significance of evolved levels of biological composition in particular. Wimsatt $(1972,2007)$ largely grounds his analysis on compositional levels, but also focuses on the causal significance of levels. In ecology, O'Neill et al. (1986) highlight the importance of time scale in distinguishing levels, though they variously appeal to spatial scale and composition as well. Craver and Bechtel (2007) 
and Craver (2007) conceive of levels as based on mechanisms and their components. Some influential philosophical discussions of levels of explanation have instead ordered levels by realization relationships and, relatedly, abstractness of description (e.g. Fodor, 1974; Putnam, 1975; Sober, 1999). Reductionists and antireductionists about explanation alike have tended to presume that these relations track compositional relationships (see Potochnik, 2010).

In this section, my aim is to explore the feasibility of a more precise levels concept that is immune to criticisms of ambiguity and related difficulties of the broad, intuitive concept of levels of organization. We can use these various ideas about levels to articulate a general problem space, a range of possibilities for where we might locate a workable levels concept. Options include the differentiation of levels with regard to part-whole composition, spatial scale, temporal scale, mechanistic composition, realization, and/or abstraction. Another approach, illustrated by Simon (1962), would be to use specific scientific theory-for instance, regarding major evolutionary transitions to more complex forms of life - to inform an articulation of levels that then has broader relevance beyond the scientific theory on which it is based.

It seems that an advocate of an unambiguous and generally applicable levels concept will need to choose among these ways of differentiating levels. When we look closely, we can see that these modes of differentiating levels don't always or even often yield the same results. Rather, composition, spatial scale, temporal scale, mechanism, realization, and abstraction relations define different organizational schemes that only partly and haphazardly overlap with one another. I briefly support this claim here; for more detail, see (Potochnik, 2017, $\S 6.2 .2)$.

Notice first that spatial scale and compositional levels are independent. While wholes and their proper parts are larger and smaller respectively, this scale relationship need not hold for the parts of different wholes at the same compositional level. Further, different parts of a given whole may be at the same compositional level but need not be similar in size. Different 
parts of a single whole can differ radically in size: large, lumbering organisms and individual waste particles are both important parts of the same ecosystem. And an entity can be a proper part of wholes of radically different sizes: a sodium ion may be an independent part of elephant or in a simple saline solution. (Thomas Reydon has pointed out in correspondence that spatial scale seems to relate more closely to compositional relationships among subatomic particles.) Spatial and temporal scales also vary independently of each other. Creep in an individual solder joint of an old lead water pipe occurs very slowly (small spatial scale, large timescale), but the catastrophic failure of an entire water distribution system resulting from cumulative solder creep is a sudden event (large spatial scale, small timescale). For similar reasons, temporal scale also doesn't relate neatly to compositional relationships.

Now consider where mechanistic organization fits in all of this. As Craver and Bechtel (2007) and others have made clear, mechanistic composition is distinct from part-whole composition. Material parthood doesn't guarantee mechanistic parthood, and the same component can participate in different mechanisms. What about the relationship between mechanistic organization and spatial and temporal scale? Mechanisms and their components do, it seems, obey a spatial ordering in much the same way material parts and wholes do. Further, the kinds of processes parts undergo that contribute to directly to action of a mechanism must occur at a faster timescale than the action of the whole mechanism, insofar as they constitute elements of the mechanistic activity. But these are only some of the processes or changes that mechanisms and their parts undergo. Other changes need not obey any such temporal ordering. For example, a gene complex involved in some trait expression will evolve very slowly, much more slowly than the timescale on which the trait is expressed in any given organism. Also similar to the relationship between part-whole composition and scale, there is no basis for spatial or temporal comparison among components or across mechanisms (Eronen, 2013). One component may be much larger or smaller or act much 
more quickly or slowly than another; distinct mechanisms may be of virtually any size and act at any of a variety of speeds.

The realization relationship, as philosophers have discussed it, does not follow partwhole composition or scale relationships. Because of multiple realization, some properties of interest may be realized by any number of different kinds of entities of different sizes: using Fodor's (1974) classic example, monetary exchanges may be realized by a stack of dollar bills or the movement of electrons. And, because of what I have called complex realization (Potochnik, 2010, 2017), there's no reason to think that the properties of parts alone realize some property of a whole. That a fish is camouflaged, for example, is jointly realized by a number of properties of the fish, its immediate environment, and its potential predators.

Finally, unlike all the other organizational schemes I've surveyed here, level of abstractness or specificity is a representational rather than metaphysical relation. As such, it's fully distinct from all of the above concepts. It is true that if you represent only big things, long time periods, and general functional relationships, you omit lots of details. Abstract representations can be of high levels in one or more of these other senses. But you also omit many details if you represent only immediate interactions among microphysical entitiesincluding, among other things, how those relate to big things, long time periods, and general functional relationships. Ultimately, you can have more or less abstract representations of anything: objects of any size, changes at any timescale, and properties structurally or functionally specified.

Because none of these different relations sometimes used to define levels relate to each other in consistent ways, any attempt to identify a precise formulation of the levels concept will need to identify one relation among these on which to base levels. To make the levels concept precise, then, we could consider choosing one of these organizational schemes as the sole basis for articulating levels. I'll next explore the prospects of that strategy. But first I want to propose a few ground rules. 
Any concept we select should do at least some of the basic work we expect of levels of organization. First, I take it that this minimally includes providing a basis for levels comparisons across entities. For this, we need to be able to show how some entity should be articulated into levels, and we also need to be able to show across different entities (or properties, processes, etc.) how these levels boundaries align with one another. Without the latter, levels comparisons across individual entities is impossible, and this has been fundamental to the invocation of levels. Second, it also seems like we want our revised levels concept to make judgments that at least loosely correspond to how levels of organization have customarily been understood. If this is a revision of our intuitive levels concept, it should work in at least some ways similar to that intuitive concept. Third and finally, when we have a handle on the features of this revisionary concept of levels, we'll need to set aside any anticipated or desired implications that we can no longer reasonably expect to obtain in light of our conceptual refinement. If a precise levels concept can be worked out, it has no claim to the various significances our prior, ambiguous idea of levels was taken to have.

So, let's survey our candidate organizational schemes with an eye to (1) the prospects for articulating levels across different entities, (2) how well the scheme coheres with traditional invocations of levels, and (3) what implications of levels we'll need to give up because of this conceptual refinement.

Part-whole composition provides an obvious candidate for the articulation of levels: any whole is at a different level from its proper parts. But, a difficulty immediately emerges. Are the waste particle and the elephant that (separately) partly compose the same ecosystem at the same level as each other? (See Eronen's chapter in this volume for additional discussion of this question.) How about the sodium ion and liver that (separately) partly compose the same elephant? If the answer to these questions is yes, then different waste particles (and different sodium ions, and so on) are on different levels from one another depending on what they compose, since these can be freely participating parts of an entity or of a part of that 
same entity (or something else entirely). If we articulate levels following simple composition relationships in this way, then we run amok of suggestion (1) I made above, that kinds of entities (or properties, processes, etc.) should be distinguishable into levels. Individual sodium ions, waste particles, and all the rest can occur at different levels depending on what sort of entity they happen to compose. An individual sodium ion (etc.) may even change level over time. This, I take it, is a counterintuitive outcome.

If instead we don't hold that all proper parts of a whole are on the same level, one level lower from the whole, then why not? What's the basis for distinguishing which parts qualify as one level down? Perhaps we expect wholes on the same level to be composed of the same kinds of parts, or parts with similar properties to one another. Again considering the elephant, a common suggestion for the next compositional level down is organs, which are in turn composed of tissues, tissues composed of cells, etc. But if wholes on a given level should be composed of the same kinds of parts, then because lots of multicellular organisms don't possess organs, the next level down from multicellular organisms simply would be cells. Organismal organization turns out to be a lot flatter than expected. And this problem recurs; for instance, are eukaryotic and prokaryotic cells on the same level? If so, organelles are not a distinct level from eukaryotic cells. This also violates common expectations. If not, then types of cells are partitioned into incommensurate systems of levels. Things quickly get messy, and violate my suggestion (2) above by deviating significantly from traditional invocations of levels.

No matter which choices are made on inclusivity or exclusivity of level membership, an organizational scheme based on composition looks less like well-behaved levels than we might hope. Either the same types of entities occupy different levels and potentially change level over time depending on what they compose, or we are left with rather unintuitive, partial ordering systems with incommensurate levels in different kinds of systems. Partwhole composition is very good at yielding a nesting relationship by dividing a type of 
entity into the types of parts that compose it. It is less good at showing how these nesting relationships relate to one another, and so less good at yielding a leveling relationship.

One way out of this morass is to turn to scientific theory to help us articulate levels. For example, major transitions in evolutionary history have been suggested as a basis for articulating compositional levels, perhaps most famously by Simon (1962). In this case, prokaryotes are on the same level as organelles, eukaryotic single-celled organisms on the same level as cells in multi-cellular organisms, and multi-cellular organisms in a class of their own. I grant that this way of conceptualizing the types of lifeforms may be enlightening for some specific inquiries. But the general significance of this ordering - even in biology - is extremely limited. For example, it is useful for developmental biologists to employ an alternative ordering based on development processes, according to which tissues and organs are each distinct levels intermediate to cells and multicellular organisms. The obvious distinction between evolutionary levels and developmental levels is regularly missed, and the significance of evolved levels for other phenomena regularly overstated. This includes by Simon (1962) himself, who stresses evolved levels even as he counts tissues, organs, and organ systems as levels.

Thus, the approach of basing compositional levels on particular scientific theory does not yield a single conception of levels of organization so much as a recipe for the construction of highly specific conceptions to suit particular inquiries. Such a highly specific levels concept is unproblematic and perhaps may be useful in the specific domain of the theory on which it is based. But, this limited scope of applicability seems (to me) to violate our expectations for a levels concept rather significantly. And, this also leads to high risk of overextension, tempting scientists and philosophers to violate suggestion (3) I made above, of giving up unearned expectations for a refined levels concept. The domain of relevance must be policed to avoid overextension and conflation of different levels concepts that give rise to different orderings, as illustrated with evolutionary and developmental levels. As 
things stand, the evolutionary levels concept in particular has regularly been overextended, generating scientific and philosophical error in the process. This risk of overextension also limits the predictive and explanatory value of these posited theory-specific levels. More than anything, they simply summarize on aspect of our theory in some domain.

Part-whole composition didn't fare well as the basis for a more precise levels concept. Now on to mechanistic levels, taken by many to be the most promising approach to levels currently available. The difficulties here mirror the problems with compositional levels. Eronen (2013) shows compellingly that mechanistic composition provides a way to distinguish nested subcomponents, components, and mechanisms, but lacks a way to create levels comparisons across different mechanisms or even different components of a given mechanism. Consequently, similar to the plight of the sodium ion above, the same entities that participate in different mechanisms will be classified as being at incomparable or potentially different levels as themselves. Further, DiFrisco (2017) suggests that mechanistic composition runs into trouble distinguishing levels above the organismal level, where (I take it) the very idea of participating in a mechanism applies in a less clearcut way. Much like classical composition, mechanistic composition is good at yielding nested hierarchies but insufficient for generating anything like traditional levels of organization. It's also the case that mechanistic composition is relative to a particular scientific investigation, perhaps more than or at least more explicitly than material composition is, as the research focus helps guide which causal processes in any given entity are under consideration. This compounds the difficulty of not being able to generalize categorization into levels across mechanisms, as a levels articulation in one domain may have no significance for another.

Let's turn next to temporal scale. DiFrisco (2017), taking inspiration from Wimsatt, systems theory, O'Neill et al. (1986), and others, advocates timescale as an alternative basis for the articulation of organizational levels. He emphasizes that hierarchical segregation is a way of preventing dynamical interaction between sub-systems of a system, which stabilizes 
the different levels of sub-systems and sub-sub-systems within a system. This is evocative of Simon's (1962) discussion of levels, where modular levels are taken to be required for the evolution of complex forms. DiFrisco's organizational scheme applies only to biological systems. It also applies only to highly limited kinds of phenomena experienced by those biological systems, namely the physiological processes that maintain homeostasis. It is thus most naturally interpreted as resulting in a hierarchical ordering of processes of those types, not of the entities that participate in those processes. As DiFrisco appreciates, this conception of levels is a significant departure from traditional invocations of levels, and it is highly circumscribed in its applicability. Indeed, he points out that "a process rate has no significance except in relation to other process rates within the same local interactive context."

Notice two things about this limitation. First, this is yet again a highly specific levels concept with a very limited domain of applicability, so it is again at risk of overextension. Recall my suggestion (3) from above, the need to recognize the intended implications we'll have to give up if they don't follow from our precise refinement of the levels concept. Second, that process rate is only significant in relation to other related process rates indicates that this intended levels concept again gives rise to nests rather than levels. Much in the way of mechanistic "levels," there is no grounds for comparison across different processes, even processes the very same entities undergo, thus falling short of suggestion (1).

Here is a candidate for a levels concept arising from spatial scale. Inspired by O'Neill et al. (1986) and more recent investigations in macroecology, Potochnik and McGill (2012) suggest articulating "quasi-levels", acknowledged to be dependent on what phenomena are under investigation and what features are of interest to the investigation. On this approach, the articulation into distinct levels across the gradient of spatial scale is based on the empirical discovery of boundaries for the dominance of different causal processes. That is, if one causal influence predominates at a given scale, and another at a higher or lower scale, these 
are at different levels. An example is the different influences on species dispersal. McGill (2010) suggests that this is controlled by random chance at the scale of a few meters, by species interactions at a scale of roughly a kilometer or so, by habitat preferences at tens to hundreds of kilometers, and by climate at a scale approaching continental size. A similar approach to levels also could be employed across timescale rather than spatial scale (giving rise to different levels schemes, and ones that are still dependent on what phenomena are investigated and which features are of interest).

This conception of levels is significantly pared down. It is explicitly dependent on focal phenomena and investigative context. Further, this articulation of levels cannot guide causal attribution but rather follows from causal attribution. One might reasonably wonder how much is to be gained from this beyond the simple recognition that causal processes vary across scales. That said, it is worth noting that something like this conception might help motivate more specific levels concepts, like evolutionary levels, developmental levels, and perhaps DiFrisco's suggested hierarchical ordering for homeostasis-preserving processes. Note that this attempt at levels again leaves us without an ability to articulate levels across entities or processes - in this case, this is a direct consequence of the ordering relating to the phenomena investigated and features of interest.

To complete our survey of potential levels concepts based on each of the different relations sometimes thought to be bound up with levels, let's briefly consider the prospects of realization and abstractness used to define levels of organization. Neither is, I think, a promising candidate. Because neither of these orderings correspond in any systematic way with compositional levels, as discussed earlier in this section, realization relationships or relative abstractness used alone yields nothing remotely approximating the levels concept as it has typically been invoked. As discussed above, realization relationships do not track compositional or scale relationships. At best, this scheme would also result in nesting relationships akin to (but not aligned with) mechanistic organization. But even that might 
be out of reach, for multiple realization and complex realization significantly complicate the relationship between realizers and what is realized. Quite many properties will jointly and variously contribute to realizing any given property, and once realization is dissociated from material composition, there is no reason to expect size ordering or containment of realizers and what they realize.

As for abstractness, I suggested above that levels of abstraction are representational rather than metaphysical. Floridi (2008) calls these "epistemological levels" and explicitly distinguishes them from levels of organization, arguing that though the latter are untenable, the former are useful. He considers Marr's three levels of analysis to be a prime example of levels of abstraction. Marr (1982) distinguishes among the computational, algorithmic, and implementational levels of description for cognitive processes. Notice that even this very different approach to levels has some of the same limitations as the others we surveyed above. Marr's levels scheme in particular applies only to a very specific kind of phenomena, i.e. cognitive or information-processing systems. And as Floridi details, they have regularly been conflated with other, very different levels concepts. Like evolutionary levels, then, this levels scheme is motivated by specific scientific theory, and its usefulness is correspondingly constrained by the applicability of the theory, as well as whether and in what form the theory is maintained. So, for example, it may turn out that computational models of cognition are of limited value, and this would undermine the significance of that representational level.

Most important for my purposes, a conception of levels in terms of abstraction bears little similarity to levels of organization as they have typically been invoked. We have already explored how abstractness varies wholly independently from composition and scale: anything large or small can be represented in more or less detail. Levels of abstraction may be a useful conceptual tool in some scientific pursuits, but that is of no help in making good on an ontological or metaphysical levels concept. And, as with Marr's levels of abstraction, it has been common to conflate representational levels of abstraction with compositional 
levels of organization. It's also worth pointing out that, like the other relationships I have surveyed, abstractness relationships tend to characterize nests rather than levels. This is because any given representation can be made more specific, less abstract, by incorporating any of a range of different details. It is thus quite easy to generate incommensurate levels of abstraction by adding or removing different ranges of details.

In this section, I first showed that all of the different relationships sometimes taken to be bound up with levels of organization systematically deviate from one another, thereby contributing to the ambiguity of the levels concept. I then explored the prospects for each of those relationships to be used alone as the basis for articulating levels of organization. I believe this survey has revealed no promising general conception of levels of organization. To be clear, it's not that there is a bit of messiness in levels divisions or an occasional failure, but the inability to ground any systematic ordering scheme of levels. Clear themes have also emerged in the limitations of each of these candidates for the basis of a levels concept.

Recall my specification at the outset that we should attend to (1) the prospects for articulating levels across different entities, (2) how well the scheme coheres with traditional invocations of levels, and (3) what implications of levels we'll need to give up because of this conceptual refinement. It turns out that every relationship surveyed fails on (1), articulating levels across different entities, thus giving rise to an ordering scheme not of levels but with more resemblance to nests. This is a significant shortcoming, as it gets at the very heart of the levels concept. Moreover, if all attempts to render the levels concept precise suffers from this same difficulty, then any levels concept invoking several of these relationships will likely suffer from the same problem. As for (2), realization relationships and abstractness by themselves bear no similarity to traditional expectations for levels of organization. Other ordering schemes, such as levels in temporal scale, deviate less radically from traditional expectations, but still deviate markedly. And, I suggested for part-whole composition that the only way of solving problems with (1) result in a very flat hierarchy of levels that departs 
significantly from traditional expectations.

And then there is the question, (3), of what implications of levels we'll need to give up due to a conceptual refinement in what levels of organization are. The most promising candidates we have seen for a levels concept are highly domain-specific and thus interest-relative. Such a highly local approach to levels is explicitly motivated by Love (2012), who focuses on the example of the four levels of protein structure. Similarly, one may emphasize evolved levels, for example, due to what we understand of the major evolutionary transitions. But, as I have suggested, domain-specific and interest-relative levels have little to no implications for other biological processes. Evolved levels should not be expected to have significance for present structural organization, development, nor even for what entities are now subject to evolutionary forces. Similarly, a conception of levels based on spatial scale will give rise to very different levels systems for different phenomena, and levels in the temporal scale of homeostatic processes have no implication for any other kinds of processes.

Furthermore, even for highly specific formulations of levels, articulating well-defined levels may still be a challenge. Protein-folding, Love's (2012) example, may be an instance where there is clear uniformity in structure and domain of applicability such that levels can be distinguished across entities. But this is more challenging for other, similarly localized levels articulations. Structural organization in many other entities does not involve this manner of discrete stages. And evolved levels may be better understood as simply the historical introduction of novel forms of evolutionarily relevant integration rather than levels of structural organization at all. It may well be that for some circumscribed scientific pursuits, like protein folding, there is a workable conception of levels of organization. But, I suspect 'levels' is not the most apt description of many instances of local organization. And, with any successful localized levels concepts, we must strenuously avoid conflation or usage slip among them or with a more general conception of levels, the promise of which we have not been able to make good on. This task is made more difficult by the fact that 
both of these forms of conflation and usage slip are historically and currently overwhelmingly common in appeals to levels.

\section{Heuristic Use of the Levels Concept}

We have explored the prospects for refining our concept of levels of organization into a more precise and defensible version and come up short. So, let's now consider the other kind of strategy to defending the usefulness of our levels concept. In the previous section, I distinguished among the various features levels have been taken to have, to show that they don't hang together, and to show that none is by itself promising as a basis for well-founded levels of organization. But, you might think, this is common for our concepts, commonsense and scientific alike. There are often limitations in the domain of a concept's applicability, just as evolutionary and developmental levels are applicable to different biological phenomena. Concepts can also be usefully ambiguous, as the gene is defined in different, inequivalent ways in different scientific contexts that are nonetheless useful to lump together. Indeed, the philosopher Otto Neurath thought vague, ambiguous concepts - what he called Ballungenwere by necessity at the basis of scientific inquiry (Neurath, 1983; Cartwright et al., 1996; Potochnik and Yap, 2006). Perhaps, then, equivocation among distinct relationships is not a weakness of the levels concept but a strength. As many have emphasized, including Brooks in this volume, biology textbooks are organized around the concept of levels of organization. So, in line with this use, might levels of organization at least be a productive heuristic?

It seems Wimsatt has something like this in mind. He sees levels as related to most of the features I identified in the previous section: composition, evolved organization, spatial and temporal scale. But he also acknowledges deviation from the alignment of these features, and he anticipates phenomena, "causal thickets", that do not feature levels at all. Inspired in part by Wimsatt, some other recent levels proponents more explicitly advocate this view. 
Brooks (2017) demonstrates the variability of the levels concept and defends this as a strength of the concept. And, in another paper, Brooks and Eronen (2018) explicitly advocate the heuristic use of levels, a use that they think relies on the term's polysemy. They say, "we offer an approach that... embraces the ambiguity of levels as vital to the flexibility the concept exhibits in expressing many distinct ideas." Brooks and Eronen hold that the levels concept is useful in structuring scientific problems by suggesting how a phenomenon can or should be studied.

This seems like a promising strategy to accommodate a scientific concept that eludes precise definition. It may be impossible to give a global definition of levels of organization, and the usefulness of the term may vary. But, there's no denying that many scientists and philosophers have invoked levels terminology in a variety of projects. And levels play a fundamental role in biology education. So accepting variability in the meaning of levels, along with some limitations and inconsistencies, may be the best way forward.

Unfortunately, though, I'm not convinced that advocating a heuristic levels concept has much better prospects than our search for a precise concept of levels of organization. I'll outline four concerns; the first regards the value of this heuristic. This concern follows from the dead end at which we ended up in the previous section's search for a precise levels concept. There I described how the different features the levels concept is expected to have in fact come apart from one another, and how none of those features is particularly well suited as a basis for articulating general levels of organization. All of the relationships under consideration produced orderings more like nests than levels and were in other ways significantly out of step with what has traditionally been said about levels. Contrast this to gene concepts. These differ in their import and applicability across projects in biology, but gene concepts in one form or another have been central to a number of theoretical and empirical projects. There is some variability in what we call a gene, but genes are inarguably biologically central. I don't see evidence that such a thing can be said of any combination of 
the levels concepts we have surveyed. It's one thing for a heuristic to vary in its applicability and usefulness, but I am concerned that there is no scientific investigation in which this heuristic is more apt and useful than misleading.

The examples Brooks and Eronen (2018) provide of the usefulness of levels in structuring scientific problems are an opportunity for me to illustrate this point. One of their examples is the use of levels to separate a system into its component parts and then relate those back to the whole phenomenon to which they belong. They have in mind localization and decomposition of the kind Bechtel and Richardson (1993) emphasized. I agree that this can be useful for some scientific projects. But, I wonder, is any levels concept important for such a project? To the contrary, this simply seems to be a matter of exploiting part-whole or mechanistic composition when such composition is important to the phenomenon under investigation. Material or mechanical composition is certainly important to this kind of a project, but I fail to see how the articulation of levels is doing any work beyond this. One can, of course, investigate parts and wholes without delineating levels of parts and wholes.

Another of Brooks and Eronen's examples is the identification of levels to support moving scientific inquiry to another level in order to gain insight into a problem or to generate hypotheses for solving that problem. They suggest this can involve "looking to new things (i.e. natural objects) or looking to new epistemic resources (descriptions, methods, models)". Brooks and Eronen appeal to systems biology's consideration of dynamics in a whole system to illustrate this idea. I also grant the usefulness of this strategy: considering new influences or methods can be scientifically fruitful. But, here too, I don't see what work is done by levels per se. These authors emphasize the historical importance of appeals to levels in systems biology's resistance of reductionism. I agree that some of the appeal of a levels concept is this historically important role. But one can just as well resist reductionism - and be creative about where in nature we look and what methods we apply - without positing that nature is organized in levels. So, granting Brooks and Eronen (2018) the potential value of both of 
these strategies, that value does not provide evidence of the usefulness of the levels concept per se.

The first concern I identified with a heuristic levels concept is that there's not clear evidence of its value. My second concern is that I fear this heuristic invites overextension and overly literal interpretation. The recent history of scientific and philosophical work is littered with instances of scientists and philosophers conflating different levels schemes and presuming the strict reality of levels. Here are just a few examples. Simon (1962) emphasizes evolved levels even as he discusses development. Craver and Bechtel (2007) claim that mechanistic levels are a species of compositional, or part-whole, levels (though later work by both authors explicitly distinguish these levels schemes). And, evocative of earlier reductionist views of levels in philosophy, ecologist Lidicker (2008) describes the universe as "composed of hierarchically arranged systems" and biological systems as comprising the "pyramid of life." Examples also abound of scientists and philosophers overselling the significance of levels, anticipating metaphysical, epistemic, explanatory, and causal significance that fails to obtain (see Potochnik and McGill, 2012). In the face of the difficulties with making good on the levels concept identified earlier in this paper, simply endorsing the heuristic use of the levels concept invites such overextension and overly literal interpretation.

Here's a third worry. Beyond limited value and risk of overextension and overly literal interpretation, I also think the levels heuristic may systematically mislead. Despite all the strong claims to the contrary, and all the smart people who part ways with me on this, I wonder whether our world might just not be aptly described as ordered into levels. I am supposed to be impressed by pockets of well-ordered behavior seemingly insulated from external fluctuations, by components of systems capable of largely independent action, and by the extent of similarities among complex forms and the implications of those similarities. But instead, I am shocked by how often external influences shape local behavior (and in ways that tend to escape our notice), how variable the action of components of a system 
often turn out to be and how subject to top-down control, and by the extent of difference among complex forms and the infinite variability produced by evolution and development. I realize that I am an outlier in my ideas about levels of organization (though see Thomasson, 2014). Perhaps it's best for me to leave this point with an invitation for responses to the following question: Why should I believe that the levels heuristic is apt - that our world, or significant swaths of our world, is hierarchically structured?

This brings me to the last concern I want to raise with the heuristic use of the concept of levels of organization. I wonder whether there aren't other, better heuristics that looking through the lens of a levels heuristic has occluded. I suspect there is a broad human tendency, perhaps in part due to the nature of our sensory systems, to focus on the significance of factors that operate on our spatial and temporal scales and to look to the parts of a system to explain its behavior. If this is not a human tendency in general, it has at least been a tendency in much of the history of science to date. Particularly in light of the foundational role that the concept of levels of organization has played in biology education and elsewhere, the levels heuristic may contribute to reinforcing these expectations of our world. Yet these expectations are, I believe, regularly violated. If this is right, then maintaining levels in a heuristic use enables us to postpone the recognition of other heuristics, heuristics that are potentially more apt and more productive either across the board or in particular scientific domains.

Here are a few candidates to illustrate the idea. Perhaps the concept of networks would be a promising alternative heuristic to explore. This relates to familiar technological innovations that might be used to disrupt our expectation that local influence is the only form of important influence and to help us anticipate large-scale influences and top-down coordination. A related notion is that of causal complexity (see, among others, Potochnik, 2017). This is less familiar and less evocative, but it does anticipate open-ended lists of causal relationships traversing spatial and temporal scales, causal interaction and nonlinear 
behavior. All of these are, I suspect, more common than causal relationships well-ordered by levels of organization. Here's an idea for a heuristic organizational scheme - or rather two schemes - to replace the levels heuristic, inspired by Eronen (2015). The levels heuristic anticipates that scale and composition go together, but as we have seen, they do not. But these two customary ingredients of the levels heuristic might be used independently of each other and of levels to categorize the range of phenomena in our complex and variable world. Scientists describe relationships that are scale invariant and those that are scale dependent. Similarly, composition is sometimes of crucial relevance and other times nearly irrelevant. Exploring the extent to which phenomena are scale bound or not and compositionally dictated or not would be a radically different, open-ended approach to categorizing our world.

None of these alternative heuristics requires setting aside the levels heuristic. But it seems likely that viewing the world through the lens of levels - organizing textbooks and philosophical and scientific inquiry according to the expectation of levels - has led us to recognize and emphasize some features of the world to the exclusion of others. Scientific discovery and philosophical breakthroughs have, I think, increasingly made clear that deep features of our world deviate from the expectation of well-ordered levels. These features are rendered less visible and taken less seriously when they are merely viewed as exceptions or limitations to an overall architecture of levels.

My concerns with preserving the concept of levels of organization as a heuristic are, thus, the limited value of this heuristic, the likelihood that the heuristic is overextended and interpreted too literally, the possibility that the heuristic of levels systematically misleads us about the nature of our world, and filling conceptual space that, given all of these limitations, might be better filled by a competing heuristic. Science is filled with simplifications, polysemic concepts, and heuristics. The question I am urging is whether levels of organization is one that earns its keep. Perhaps the basis for our levels concept turns out to be simply 
our impulse to categorize the variety we encounter in our world and to identify similarities among the various resulting categorizations. If this is right, calling the levels concept a heuristic is not simply an acknowledgement of the necessary variability and ambiguity in scientific terminology, but a fallback position adopted when one is faced with the failure to make good on the idea that our world is organized into levels.

\section{What's to Lose with Levels?}

In this paper, I have surveyed two approaches to a concept of levels of organization: rendering it precise and advocating its heuristic use. I have worked through problems that I believe face each approach. It's worth pointing out that, because of the nature of these problems, the pursuit of both strategies simultaneously in philosophical and scientific literatures compounds the difficulties facing each. Attempts to identify a precise refinement of the levels concept tempts us to think there is a way to make good on the heuristic, and thus to take the heuristic too literally. And then, the heuristic use of the levels concept tempts us to think that we don't need to fully work through a precise refinement of the levels concept, obscuring the conceptual difficulties it faces. That both kinds of approaches are advocated is, I think, evidence of the problems plaguing the concept of levels of organization.

Now is the point in the paper to ask, "so what?" What would I wish to be done? First and most basically, I urge wider recognition of the variety, dissociation, and limitations of levels concepts. If "levels" is polysemic, we should at the very least track the polysemy to avoid scientific error. This is made especially difficult given that some philosophers are advocating conceptual refinement while others are advocating broad heuristic use. Part of this project should be to carefully distinguish metaphorical from literal applications of the concept and to be aware of the domain within which any given use of the concept applies. For example, as I said above, that more complex forms have evolved at the major 
transitions of evolutionary history is not reason to expect those ancestral forms to have special significance for biological organization today, such as development. And yet, though it's now well appreciated that ontogeny does not recapitulate phylogeny, evolutionary levels are still invoked in developmental contexts. Close on the heels of this point is a second idea: scientists and philosophers should investigate the significance of levels in a given domain rather than draw conclusions about that domain from the presumed significance of levels. Some bits of our world may turn out to be organized into levels, at least in certain respects. But given the complex variability of our world, that form of organization cannot be presumed.

Third and finally, scientists and philosophers should actively try to cultivate alternative heuristics - networks, causal complexity, scale invariance, and others. Try for a moment to imagine what organizational schemes are available for biology textbooks other than compositional "levels", and how these different schemes might differently educate our biologists. What about, for example, a textbook ordered by the spatial scale and temporal scale over which different influences tend to dominate? Or a textbook that begins with a figure depicting the interdependence of phenomena, and is then ordered by the specific research questions that have motivated a focus on different features of these complex phenomena?

In my view, our adherence to the levels concept in the face of the systematic problems plaguing it amounts to a failure to recognize structure we're imposing on the world, to instead mistake this as structure we are reading off the world. Attachment to the concept of levels of organization has, I think, contributed to underestimation of the complexity and variability of our world, including the significance of causal interaction across scales. This has also inhibited our ability to see limitations to our heuristic and to imagine other contrasting heuristics, heuristics that may bear more in common with what our world turns out to actually be like. Let's at least entertain the possibility that the invocation of levels can mislead scientific and philosophical investigations more than it informs them. I suggest that 
the onus is on advocates of levels of organization to demonstrate the well-foundedness and usefulness of this concept.

\section{Acknowledgments}

Thanks to Daniel Brooks, James DiFrisco, and Bill Wimsatt for coordinating a productive and fun workshop and to the Konrad Lorenz Institute for hosting. I learned so much from the other participants, and I had a great time in the process. Thanks also to Thomas Reydon and Markus Eronen for helpful comments on an earlier draft of this chapter. The chapter also benefitted from audience feedback at talks in the Department of History and Philosophy of Science at the University of Pittsburgh and the Department of Philosophy at the University of Colorado, Boulder.

\section{References}

Bechtel, William, and Robert C. Richardson (1993), Discovering Complexity: Decomposition and Localization as Strategies in Scientific Research, Princeton: Princeton University Press.

Brooks, Daniel S. (2017), "In Defense of Levels: Layer Cakes and Guilt by Association", Biological Theory 12: 142-156.

Brooks, Daniel S., and Markus I. Eronen (2018), "The significance of levels of organization for scientific research: A heuristic approach", Studies in History and Philosophy of Biological and Biomedical Sciences : online first.

Cartwright, Nancy, Jordi Cat, Lola Fleck, and Thomas E. Uebel (1996), Otto Neurath: Philosophy Between Science and Politics, Ideas in Context, vol. 38, Cambridge: Cambridge University Press.

Craver, Carl F. (2007), Explaining the Brain: Mechanisms and the Mosaic Unity of Neuroscience, Oxford University Press. 
Craver, Carl F., and William Bechtel (2007), "Top-Down Causation Without Top-Down Causes", Biology and Philosophy 22: 547-563.

DiFrisco, James (2017), "Time Scales and Levels of Organization", Erkenntnis 82: 795-818.

Eronen, Markus I. (2013), "No Levels, No Problems: Downward Causation in Neuroscience", Philosophy of Science 80: 1042-1052.

— (2015), "Levels of organization: a deflationary account", Biology and Philosophy 30: $39-58$.

Floridi, Luciano (2008), "The Method of Levels of Abstraction", Minds \& Machines 18: 303-329.

Fodor, Jerry (1974), "Special Sciences: The Disunity of Science as a Working Hypothesis", Synthese 28: 97-115.

Lidicker, Jr., William Z. (2008), "Levels of Organization in Biology: On the Nature and Nomenclature of Ecology's Fourth Level", Biological Reviews 83: 71-78.

Love, Alan C. (2012), "Hierarchy, Causation and Explanation: Ubiquity, Locality and Pluralism", Interface Focus 2: 115-125.

Marr, David (1982), Vision. A Computational Investigation into the Human Representation and Processing of Visual Information, W.H. Freeman and Company.

McGill, Brian J. (2010), "Ecology. Matters of Scale.", Science 328: 575.

Neurath, Otto (1983), Philosophical Papers 1913-1946, Vienna Circle Collection, vol. 16, Dordrecht: D. Reidel Publishing Company.

O’Neill, R. V., D. L. DeAngelis, J. B. Waide, and T. F. H. Allen (1986), A Hierarchical Concept of Ecosystems, Princeton: Princeton University Press.

Oppenheim, Paul, and Hilary Putnam (1958), "Unity of Science as a Working Hypothesis", in Herbert Feigl, Michael Scriven, and Grover Maxwell, eds., Minnesota Studies in the Philosophy of Science, Minneapolis: University of Minnesota Press, vol. 2, 3-36.

Potochnik, Angela (2010), "Levels of Explanation Reconceived", Philosophy of Science 77: 59-72. 
- (2017), Idealization and the Aims of Science, University of Chicago Press.

Potochnik, Angela, and Brian McGill (2012), "The Limitations of Hierarchical Organization", Philosophy of Science 79: 120-140.

Potochnik, Angela, and Audrey Yap (2006), "Revisiting Galison's "Aufbau/Bauhaus" in Light of Neurath's Philosophical Projects", Studies in History and Philosophy of Science 37: 469-488.

Putnam, Hilary (1975), Philosophy and our Mental Life, Cambridge: Cambridge University Press, Philosophical Papers, vol. 2, chap. 14, 291-303.

Rheinberger, Hans-Jörg, and Staffan Müller-Wille (2017), The Gene, Chicago: University of Chicago Press.

Rueger, Alexander, and Patrick McGivern (2010), "Hierarchies and Levels of Reality", Synthese 176: 379-397.

Simon, Herbert (1962), "The Architecture of Complexity", Proceedings of the American Philosophical Society 106: 467-482.

Sober, Elliott (1999), "The Multiple Realizability Argument Against Reduction", Philosophy of Science 66: 542-564.

Thomasson, Amy (2014), "It's a Jumble Out There", American Philosophical Quarterly 51: $285-296$.

Wimsatt, William C. (1972), "Complexity and organization", in K. F. Schaffner and R. S. Cohen, eds., PSA: Proceedings of the Biennial meeting of the Philosophy of Science Association, Dordrecht: D. Reidel, 67-86.

— (2007), Re-Engineering Philosophy for Limited Beings, Cambridge: Harvard University Press. 